\title{
Diffusion and Emergence in Social Networks
}

\author{
Akira Namatame \\ Dept. of Computer Science, \\ National Defense Academy,Yokosuka, Japan \\ www.nda.ac.jp/ nama
}

\begin{abstract}
Diffusion is the process by which new products and practices are invented and successfully introduced into society. Numerous studies on the diffusion of individual innovations have been conducted, many exhibiting common features such as the famous S-shaped diffusion curve. One basic question posed by innovation diffusion is why there is often a long lag time between an innovation's first appearance and the time when a substantial number of people have adopted it. An extensive amount of theoretical and empirical literature has been devoted to this phenomenon and the mechanisms behind it. New ideas, products, and innovations often take time to diffuse, a fact that is often attributed to the heterogeneity of human populations. In this chapter, we provide an overview of research examining how the structure of social networks impacts diffusion process. The diffusion process enhances innovations via feedback of information about the innovation's utility-which can be used to make future improvements - to many different users. This aspect of the diffusion process is similar to the micro-macro loop, which is an essential part of emergence. The aim of this research is to understand how the structure of social networks determines the dynamics of various types of emergent properties occurring within those networks. For emergence at the social level, patterns of social interactions are critical.
\end{abstract}

Keywords: interaction, contagion, diffusion of innovations, tipping point, threshold, consensus, synchronization

\section{Introduction}

For decades, social scientists, economists and physicists have been interested in the fundamental — and widespread - question of how infectious diseases, new technological practices, or the latest trends spread through society. When a new technology appears, society's members have the chance to become aware of the innovations of the new technology and incorporate it into their lives. The main study on diffusion modeling is based on the Bass model (Bass, 1969). The Bass diffusion model describes the process by which new products are adopted as an interaction between users and potential users. When an innovation is a product to be consumed by individuals, a single consumer can decide whether or not to adopt it.

More specifically, the Bass model formalizes the aggregate level of penetration of a new product, emphasizing two processes: external influence via advertising and mass media as well as internal influence via word-of-mouth. The decision of a consumer is described as the probability of the consumer adopting the new product at a specific time, and it is assumed to depend on both external and internal influences. The Bass model displays a cumulative $\mathrm{S}$ curve of adopters: when the number of users of a new product is plotted against time, the resulting curve shows an S-shaped distribution-adoption 
proceeds slowly at first, accelerates as it spreads throughout the potential adopting population, and then slows down as the relevant population becomes saturated. The S-shape is a natural implication of the observation that adoption is usually an absorbing state. The fast growth of diffusion is generated by the interaction between early adopters and late adopters. The Bass model, however, does not specify the consumer decision-making process or how consumers communicate with and influence one another at the micro level. The Bass model assumes the population of consumers to be homogeneous - such diffusion models are referred to as aggregate models.

Rosenberg (1972) observed two dominant characteristics of the diffusion process: the overall slowness of the process, on one hand, and the wide variations in the rates of acceptance of different inventions on the other. Empirical measurement and study have since confirmed his view. Why is diffusion sometimes slow? Why is it faster in some regions than others? Why do rates of diffusion differ among different types of innovations? What factors govern the wide variation in diffusion rates? Hall (2003) provides a comparative historical perspective on diffusion that looks at the broad economic, social, and institutional determinants. In the modern world, markets occasionally accept innovations very slowly, despite technological advances. Chakravorti (2004) also provides many examples of the slow pace of fast change.

New ideas, products, and innovations often take time to diffuse, a fact that is often attributed to the heterogeneity of human populations. Global markets are now larger and more complex than ever before. As such, the amount of information available to consumers has considerably increased; as a result, consumers must spend much time thinking and hesitating before making a decision. Thus, consumers require more time to make personal decisions about matters such as whether to use a certain new product or participate in the latest technology.

Consumers may realize different aspects of the benefits and costs of a particular innovation, have different beliefs regarding benefits and costs, hear about the innovation at different times, or delay in acting on the information they receive. Young (2007) analyzes the effect of incorporating heterogeneity into three broad classes of models: contagion, social influence, and social learning. In addition, when a consumer has many neighbors, these represent many potential sources of information, and the consumer may have problems effectively handling such a large amount of information. Clarification of this relationship calls for analysis of the types of social interactions linking various individuals within a society.

Societies consist of individuals and the social systems that largely determine how individuals behave and interact. Individuals' actions are influenced by friends, acquaintances, and neighbors, and relationships between individuals form the basis of social networks. One of the cardinal rules of human behavior is "birds of a feather flock together"- friends of friends become friends, and this property fosters the development of dense clusters of connections throughout social networks. However, little is known about the dynamic processes on networks and how these processes depend on network properties. Among research concerning dynamic diffusion processes, the diffusion of diseases has received the most attention (Colizza, 2006).

An emergent pattern or emergent property can appear when a number of simple entities operate in an environment, forming more complex patterns collectively. In this sense, many insights can be derived from the study of biology. Many biological systems appear to demonstrate emergent behavior, such as the sudden dispersion of a flock of 
animals in the presence of a predator (Reynolds, 1987). Emergent properties in biological systems appear to govern behaviors that are advantageous to the group as a whole. A natural interest in the behavior of animals and individuals, coupled with the possibility of utilizing these behaviors to design desirable systems, has prompted the study of self-organization in adaptive systems (Namatame, 2006). In adaptive systems with large numbers of entities, global behavior emerging from localized interactions is a critical concept. Understanding and shaping emergence may be essential to the survival social systems.

Emergent patterns are not the property of any single entity, nor can they easily be predicted or deduced from behavior in lower-level entities. Having a large number of interactions alone is not enough to guarantee emergent patterns - a system may need to reach a certain critical mass before it can self-generate emergent patterns. In some cases, a system must reach a combined threshold of diversity, organization, and connectivity before emergent properties appear. Unintended consequences and side effects are closely related to emergent properties. In other words, the global or macroscopic functionality of a system is the sum of the side effects of all emergent properties.

Social network structures are also related to emergent properties that cannot be trivially derived from the properties of their components. Simple rules concerning individual behavior can result in very complex phenomena at the societal level. Emergent macroscopic patterns throughout the social space can be divided into dense intra-connectivity within clusters with sparse inter-connectivity between clusters (Newman, 2003). In turn, emergent stylized facts or regularities observed at the societal level also influence individual behaviors and interactions. On the other side, individual behaviors create the social networks to which individuals belong. This bi-directional causal relationship is an essential component of the study of social complexity, including diffusion process from the viewpoint of emergence.

Understanding the relationship between the different levels at which macroscopic phenomena can be observed has been made possible by the tools and insights generated in complex network research. In particular, much is known about measuring the topological properties of social networks. Recently, a number of works have focused on the rules of network formation and the relationship between the network creation process and the structure of the resulting network (Barabasi, 2000).

We begin by discussing several examples of studies that illustrate how the structure of social networks impacts macroscopic diffusion patterns. The discussions are divided into three classes. First, we discuss progressive diffusion processes. Many diffusion processes are progressive in the sense that once a node switches from one state to another, it remains in the same state in all subsequent time steps. For this type of diffusion, we focus on the correlations between social interaction patterns and the observable transmission rate at the individual level.

The second class consists of non-progressive diffusion processes where, as time progresses, nodes can switch from one state to the other or vice versa, depending on the states of their neighbors. For non-progressive diffusion processes, we focus on decision making at the individual level. This class ties individuals to the analysis of the underlying games among a network of agents.

The third class consists of bi-directional diffusion process. In this class, individuals require proper interaction rules regulating information exchange among individuals so that all individuals in the social network eventually share the same information or 
internal states. Consensus problems have a long history in computer science and control theory (Olfati-Saber, 2007). Such problems form the foundation of the field of distributed systems since they have a tradition in systems and control theory on asynchronous agreement problems. In networks of agents, consensus means to reach an agreement regarding a certain quantity of interest depending on the state of all agents. A consensus algorithm is an interaction rule concerning the information exchange between an agent and all of its neighbors within the network.

\section{Study of Diffusion and Emergence}

Diffusion is the process by which new products and practices are successfully introduced into society. The Bass model formalizes the aggregate level of penetration of a new product, emphasizing two processes: external influence via advertising and mass media and internal influence via word-of-mouth. The Bass model assumes all consumer populations to be homogeneous - such diffusion models are referred to as aggregate models. However, an individual decision rule can also be derived from the Bass model: the number of individuals who adopt a new product at a given time is a function of the number of individuals who have already adopted the product. If we calculate the expected number of adopters at a given time, the aggregate model displays a cumulative S curve of adopters (Katona, Zubcsek \& Sarvary, 2007). The probability of an individual adopting a product, then, is a function of the number of that individual's neighbors who have already adopted the product. Naturally, if a person has more friends already using a certain product or service, he or she will be more likely to also adopt the product or service. The familiar Bass model does not formalize this complex emergent aspect of the diffusion process.

The diffusion of innovations, new ideas or rumors can be modeled after the spread of infectious diseases (Meyers, 2005). Because epidemiological models describe infection in terms of transmissibility and susceptibility, however, such models cannot adequately describe diffusion processes that usually involve individual information processing and decision-making. People are usually affected by word-of-mouth, which is referred to as a social influence. What influences people to participate in the diffusion process? How do consumers choose which items to buy? These questions are constant in the minds of many scientists in different fields. Various models of human behavior span the extremes from simple and easily influenced behavior to interdependent and complex behavior where nothing is easily controlled or predicted.

Spielman defines an innovation system as "a network of agents, along with the institutions, organizations, and policies that condition their behavior and performance with respect to generating, exchanging, and utilizing knowledge" (Spielman, 2005). An innovation system reflects one aspect of value chain analysis by bringing actors together in the application of knowledge within a value chain. This definition highlights the need for a holistic view of the nature and structure of interactions among agents linked to one another within networks. The adoption of an innovation by one agent in a network can have positive or negative impacts on the behavior of other agents, which is often unintentional or unpredictable.

The growing literature on social interactions has examined how decision-making by an agent is influenced by the decisions of others in the context of a multitude of social phenomena. Examples include studies on the diffusion of new technologies (Arthur, 1989), herding behavior in stock markets (Banerjee, 1992) and the diffusion of 
conventions and social norms (Ellison, 1993; Morris, 2000). While the research on diffusion recognizes the importance of complex interactions among agents, it rarely explains or measures unintended impacts, both positive and negative, on agents within a network. More to the point, current methodologies provide insufficient explication of the concept of network externalities. This suggests the need for alternative tools to provide a more enhanced understanding of these interactions.

In social systems that involve large numbers of interacting agents, emergent global stylized facts that arise from local interactions are a critical concept. The emergence of the Internet, for instance, marked the appearance of totally new forms of social and economic exchange (Sole, 2003). As a technological innovation, the Internet provided a new stage for communication and information processing within societies, leading to the creation of new previously non-existent system structures.

Every day, billions of people worldwide make billions of decisions about many things. The aggregation of these unmanaged individual decisions often leads to unpredictable outcomes. People constantly interact with each other in different ways and for different purposes. Somehow, these individual interactions exhibit some degree of coherence at the aggregate level, and therefore aggregation may reveal structure and regularity. The individuals involved may have a very limited view of the whole system, but their activities are coordinated to a large degree and produce desirable outcomes at the aggregate level, often exhibiting the features of emergent properties-properties of the system that individual components do not have. These emergent properties are the result of not only the behavior of individuals but the interactions between them (Ball, 2004).

Important processes that take place within social networks, such as the spreading of opinions and innovations, are influenced by the topological properties of those networks. In turn, the opinions and practices of individuals can have a clear impact on network topology, for instance, when conflicting opinions lead to the breakup of social interaction. From an applied point of view, it is desirable to compose an inventory of the types of microscopic dynamics that have been investigated in social networks and their impact on emergent properties at the network level. Such an inventory could provide researchers with specific guidelines concerning the kinds of phenomena present in social systems where similar diffusion processes are at work.

A large number of interactions is not enough to guarantee emergent patterns in a system; in some cases, a certain critical mass must be reached before emergent patterns can be generated - a system must reach a combined threshold of diversity, organization, and connectivity before emergent properties appear. Topological network properties should be derived from the dynamics by which the networks are created. Formation of new links, for example, may occur when individuals introduce their friends to new people, creating new connections between existing nodes. In reality, social networks are formed by social processes in which individuals create and maintain social relationships; in turn, these social processes influence the dynamics of social networks. This process results in the self-organization of social systems, in which social relations depend on - and, in turn, influence - the relationship between individuals and groups within society. The direction of this research to come is to understand how the structure of social networks determines the dynamics of various types of emergent properties occurring within those networks. For emergence at the social level, patterns of social interactions are critical. 
One of the major focuses of this research is the dynamics of networks. Here, the topology of the network itself is regarded as a dynamical system-it changes in time according to specific, often local rules. Investigations in this area have revealed that certain evolutionary rules give rise to peculiar network topologies with special properties. For instance, let us consider a graph $\mathrm{G}(\mathrm{n}, \mathrm{p})$ that consists of $\mathrm{n}$ nodes (or vertices) joined by links (or edges) with some probability p. Specifically, each possible edge between two given nodes occurs with a probability $\mathrm{p}$. The average number of links (also called the average degree) of a given node will be $\mathrm{z}=\mathrm{np}$, and it can be easily shown that the probability $\mathrm{p}(\mathrm{k})$ that a vertex has a degree $\mathrm{k}$ follows a Poisson distribution. This model displays a phase transition at a given critical average degree $z_{c}$ $=1$. At this critical point a giant component forms and for $\mathrm{z}>\mathrm{z}_{\mathrm{c}}$ a large fraction of nodes are connected in a web, whereas for $\mathrm{z}<\mathrm{z}_{\mathrm{c}}$ the system is fragmented into small sub-webs (Durrent, 2007).

The second major line of network research focuses on dynamics on networks. Here, each node of the network represents a dynamical system. Individual systems are coupled according to the network topology. Thus, the topology of the network remains static while the states of the nodes change dynamically. Important processes studied within this framework include synchronization of the individual dynamical systems and contact processes, such as opinion formation and epidemic spreading. Studies like these have clarified that certain topological properties have strong impacts on the dynamics of and on networks.

\section{Types of Social Interaction and Network Topologies}

Human interactions are governed by formal and informal social networks. The approach of social networks formalizes the description of networks of relations in a society. In social networks individuals can be represented as nodes, and relations or information flows between individuals can be represented as links. These links can represent different types of relations between individuals, including exchange of information, transfers of knowledge, collaboration, and mutual trust.

\subsection{Types of Social Interaction}

Here, we present a brief overview of some forms of social interaction, divided into the following three categories:

$<$ Information transmission $>$ Individuals pass information to other individuals. An individual who knows a piece of information will share this information with friends or colleagues. A form of this process may be described as a gossip algorithm (Boyd, Ghosh, Prabhakar \& Shah, 2006), the process underlying the spread of information in social groups and societies. The fundamental question is under what conditions the information will diffuse throughout society. Whether the information will diffuse throughout a society depends primarily on the probability that it will be passed from one individual to that individual's interaction partners.

Many stylized facts on the role of social network structure on information transmission are well understood in literature on the diffusion of disease. For instance, dynamics of information transmission is also a threshold phenomenon, where below a certain value the information will spread only in a limited group; however, above the threshold it will penetrate throughout the entire society. 
<Interaction with externalities $>$ Social interdependence defines another type of interaction among individuals. Social interdependence can be understood as a dependence of outcomes of one individual decision on another individual decision. Such a relationship among individuals is described as payoffs for the choices of individuals. This is usually described with the formalism of the game theory. In this formalism, the payoff of one agent depends on the choices of other agents, and, in turn, the choice of one agent changes the payoff structure of other agents.

$<$ Consensus formation $>$ Interaction between individuals does not involve merely sharing information; its function is to construct a shared reality consisting of agreed-upon opinions. In this process of consensus formation, individuals must influence one another to arrive at a common interpretation of their shared reality. Social influence is usually defined as a change in an individual's thoughts, feelings or actions resulting from the presence of others. Social influence concerns not only formation of opinions but also a variety of other social phenomena, such as learning from others and changes in attitude.

\subsection{Interaction Topology}

$<$ Global or random network $>$ All individuals have a certain probability of interacting with everyone else in the social group. Such interaction consists of interfaces such as communication through mass media, densely connected social networks, and posts on message boards, among others. Epidemic models of phenomena like the spread of diseases, rumors, or gossip belong to this class. Epidemic models have autocatalytic properties since everybody who becomes infected becomes a new source of infection.

$<$ Local network $>$ Individuals may not interact with everyone-space imposes important constraints on interaction. Individuals have the highest probability of interaction with those who are nearby. Cellular automata are the models of choice for investigating the emergence of patterns from local interactions among individuals in various spatial configurations. Individuals are arranged in a discrete space such as a $2 \mathrm{D}$ lattice, and time proceeds in discrete states. Interaction rules are local-the state of each cell depends on the state of neighboring cells in a way specified by a specified rule (Nowak, 2006).

$<$ Small-world network $>$ The path linking any two nodes has to go though only a small number of other nodes, meaning that, in principle, messages can spread throughout the entire society in very few steps. This type of network is known as a small-world network with short paths (Watts, 1999).

$<$ Scale-free network $>$ Formation of new links may happen when individuals introduce their friends to new people; by this process, the most connected individuals acquire most new links. By extension, power is distributed according to the number of links (Barabási, Albert \& Jeong, 2000). Emergent network properties do not depend on the size of the network. A small number of individuals have many links, while many individuals have few links. 


\section{Progressive Diffusion Process}

Many diffusion processes are progressive in the sense that once a node switches from one state A to another state B, it remains with B in all subsequent time steps. This type of diffusion process is progressive. The other type of diffusion is a non-progressive process in which, as time progresses, nodes can switch from A to B or from B to A depending on the states of their neighbors. In this section, we discuss the progressive diffusion process; the next two sections are devoted to the non-progressive diffusion process.

The diffusion of information, rumors, or gossip through social networks can be modeled after the spread of infectious diseases. Clearly, becoming infected is not an individual choice, and contagion in this case is not a strategic phenomenon. In the spread of a disease, some nodes (individuals) get infected initially through some exogenous sources, and consequently some of these individual's neighbors are infected through contact. There is a possibility that a given node is immune; however, if a node is not immune, then it is sure to catch the disease if one of its neighbors is infected.

The basic diffusion model is the SIR model, in which nodes are initially susceptible to the disease and can become infected from infected neighbors. Once infected, a node continues to infect its neighbors until it is randomly removed from the system. The SIR model presumes that, once infected, a node will eventually infect all of its susceptible neighbors. Infected nodes can either recover and stop transmitting the disease or die and completely disappear from the network.

The other model is the SIS model, in which nodes, once infected, can randomly recover; however, after recovery, they are once again susceptible to infection. The SIS model corresponds well with many real-world viral infections that may cause individuals to transition back and forth between health and illness.

We can view disease propagation as a dynamical birth-death process with self-recovery. An infected node i propagates the disease to another node $\mathrm{j}$ in a single step with probability $\beta$, while at the same time an infected node $i$ may recover with some probability $\delta$. The ratio of the two factors $\lambda=\beta / \delta$ defines the relative infection rate of the disease. From this, it is known that the diffusion process is governed by the threshold phenomenon, and therefore infection spreads throughout the entire network only if

$$
\beta / \delta><\mathrm{k}>/<\mathrm{k}^{2}>
$$

where $<\mathrm{k}>$ is the average connectivity and $\left\langle\mathrm{k}^{2}>\right.$ is the average of the square of the connectivity (Meyers, 2005). Therefore, higher infection rates lead to the possibility of positive infection, as do degree distributions with high variances. The rationale behind high-variance distribution is that there will be some hub nodes with a high degree of infection, which may foster the contagion, accelerating the spread of the disease.

Note that while this analysis correctly observes that highly linked nodes are more likely to become infected, the aggregate behavior does not exhibit the S-shape common to many real-world diffusion processes. Beyond these empirical studies, this analysis provides some insight into what kinds of social structures are necessary for infection to spread; however, it does not provide an accurate figure of how extensive infection will be and how network structure affects infection rate.

Given a social network topology described with the adjacency matrix among nodes and infection and recovery rates, the epidemic dynamics is also characterized from the 
relative infection rate and the inverse of the largest eigenvalue of the adjacency matrix A $=\left(a_{i j}\right)$ (Wang \& Chakrabarti, 2003). We denote the largest eigenvalue of the adjacency matrix $\mathrm{A}$ by $\lambda_{1}(\mathrm{~A})$. If an epidemic dies out, then it is necessarily true that

$$
\beta / \delta<1 / \lambda_{1}(\mathrm{~A})
$$

For homogeneous networks, the largest eigenvalue is

$$
\lambda_{1}(\mathrm{~A})=<\mathrm{k}>
$$

For star networks, the largest eigenvalue is

$$
\lambda_{1}(\mathrm{~A})=\mathrm{k}^{1 / 2}
$$

For scale-free networks with infinite variance, the largest eigenvalue is

$$
\lambda_{1}(\mathrm{~A})=\infty
$$

Therefore, the disease may spread widely throughout scale-free networks, since this type network has no threshold.

Equation 4.2 is also critical in determining how quickly a society may recover from epidemic diffusion. The number of infected nodes decays exponentially over time. While it can be very useful to identify correlations between nodes identifying causation is more complicated without the right exogenous variation.

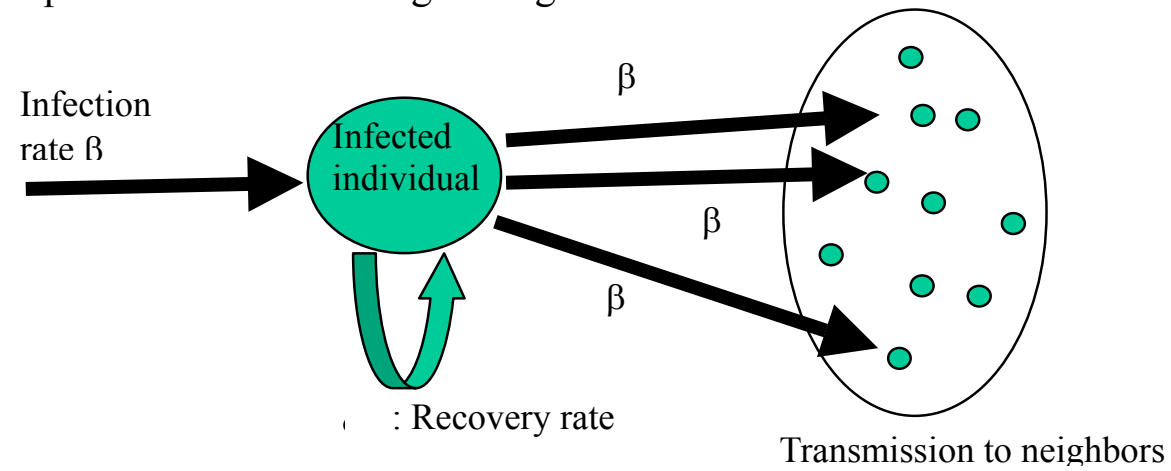

Figure 1. Basic scheme of spread of disease

\section{Non-Progressive Diffusion Process}

Epidemiological models describe infection in terms of transmissibility and recovery. These are extended diffusion models that explicitly include decisions influenced by social situations and word-of-mouth processes at the individual level (Jackson, 2008).

A fundamental question in the social sciences is to understand the ways in which new ideas, behaviors, and practices diffuse throughout human populations; for example, in the adoption of new technologies, the emergence of new social norms or organizational conventions, or the spread of languages. An active line of research in economics and mathematical sociology is concerned with modeling these types of diffusion processes as a coordination game played on a social network.

In most of the settings that form the motivation for diffusion models, coexistence is the typical outcome. An important piece, however, that is arguably missing from the 
basic game-theoretic models of diffusion is a more detailed picture of what is happening at the coexistence boundary, where the basic form of the model posits nodes that adopt A linked to nodes that adopt B.

Another big part of the research on innovation diffusion has focused on empirical studies showing the crucial elements of the structure of social interactions and on computational models that investigate patterns of innovation diffusion through social networks (Rogers, 2005).

For simple diffusion such as the spread of disease or rumors - in which a single active node is efficient to trigger the activation of its neighbors, hub nodes with many connections or random links connecting distant nodes allow dramatic diffusion as discussed in the previous section. However, not all propagation is the simple activation of nodes after exposure to multiple active neighbors. This type of cascade model is called a threshold model. Each agent has a threshold that determines the proportion of neighbors required to activate it.

Threshold models become structurally equivalent with coordination games on networks. This approach builds on work investigating the diffusion of innovations in the economics literature, which seeks to model how a new technology A might spread through a social network of individuals who are currently users of technology B. We consider several ways of capturing the compatibility of $\mathrm{A}$ and $\mathrm{B}$, focusing primarily on a model in which users can choose either to adopt A or B. We characterize how the ability of A to spread depends on its quality relative to B (Ellison, 1993).

There are many social interactions with positive externalities in which the underlying game is formulated as a coordination game with multiple equilibria. We begin by discussing one of the most basic game-theoretic diffusion models proposed by Morris (Morris, 2000). Morris provided a set of elegant game-theoretic characterizations for when these qualitatively different types of equilibria arise in terms of the underlying network topology and the quality of A relative to B (i.e., the relative sizes of $1-\theta$ and $\theta$ ). There can be a cascading sequence of nodes switching to $A$, such that $a$ network-wide equilibrium is reached in the limit; with all nodes adopting A or it may involve coexistence with the nodes partitioned into a set adopting $\mathrm{A}$ and a set adopting B.

More specifically, we describe in terms of the following technology adoption scenario proposed by Immorica (2007). Suppose there are two technologies $A$ and $B$ and agents must use the same technologies in order to communicate. There is a social network $G$ on the agents which governs who talks to whom. Each edge $(v, w)$ of $G$ plays a coordination game with strategies $\mathrm{A}$ or $\mathrm{B}$ : if $v$ and $w$ each choose $\mathrm{A}$, then they each receive a payoff of $1-\theta$ (since they can talk to each other using system $A$ ); if they each choose $B$, then they each receive a payoff of $\theta$. However if they choose opposite technologies, then they each receive a payoff of 0 , reflecting the lack of interoperability.

The binary decision itself can be considered a function solely of the relative number of other agents who are observed to choose one alternative over the other. The outcome depends on the strategy choices of all agents. Fortunately, in certain strategic situations, interactions among multiple agents can be analyzed by decomposing into the underlying $2 \times 2$ games. We consider strategic interactions in which agents are identically situated in the sense that every agent's outcome, regardless of the choice made, depends on the number of agents who choose A or B. Each agent faces a binary problem between two choices: A or B. For any agent, the payoff for a choice of A or B depends on which 
other agents choose. This strategic situation is modeled as the $2 \times 2$ games with payoffs shown in Table 1 . Note that $A$ is the better technology if $\theta<1 / 2$, while $A$ is the worse technology if $\theta>1 / 2$.

Let us suppose that all agents (nodes) initially adopt B. Thereafter, a small number of agents begin adopting new strategy $\mathrm{A}$ instead of $\mathrm{B}$. If we apply best-response updates to nodes in the network, then nodes will in effect be repeatedly applying the following simple rule: switch to $\mathrm{A}$ if the proportion of neighbors who have already adopted $\mathrm{A}$ is larger than the threshold $\theta$.

We propose a diffusion model that explicitly includes individual decisions influenced by social situations; in the model described above, agents decide according to their own preferences, but are at the same time influenced by other agents' decisions. This interdependence is described as a threshold rule (Watts, 2007).

\begin{tabular}{|c|c|c|}
\hline Others' choides & $\mathrm{A}(\mathrm{p})$ & $\mathrm{B}(1-\mathrm{p})$ \\
\hline Own choice & $1-\theta$ & 0 \\
\hline $\mathrm{A}$ & 0 & $\mathrm{~A}$ \\
\hline
\end{tabular}

Table 1. Payoff matrix of a coordination game

Next, let us consider a population of $\mathrm{N}$ agents. Each faces a binary problem between two choices: A or B. For any agent, the payoff for a choice of A or B depends on how many other agents also choose A or B. The payoff for each agent is given as an explicit function of the actions of all other agents; for this reason, agents have an incentive to pay attention to the collective decision. The payoffs for each agent choosing A or B are given as:

$$
\begin{aligned}
& U(A)=(1-\theta) p, \\
& U(B)=\theta(1-p)
\end{aligned}
$$

where $p(0 \leq p \leq 1)$ is the proportion of the neighbors' agents to choose A.

The binary decision itself can be considered a function solely of the relative number of other agents who are observed to choose one alternative over the other. The outcome depends on the choices of all agents. We have two stable Nash equilibria at the end points, where all agents choose either $\mathrm{A}$ or $\mathrm{B}$. If everyone chooses $\mathrm{A}$ or $\mathrm{B}$, no one is motivated to switch. In this case with multiple equilibria, the problem is to get a concerted choice. Since $\theta<1 / 2$, there is no ambiguity about which equilibrium is the superior one; the problem is then how to achieve the most efficient situation where all agents choose $\mathrm{A}$. The direction in which collective behavior will move depends on the point where the proportion of agents choosing $A$ is $\theta$. If only a few choose $\mathrm{A}$, they will subsequently switch to $\mathrm{B}$, and if most agents choose $\mathrm{A}$, the few agents who choose $\mathrm{B}$ will soon switch to $A$. If many agents choose $B$, no agent is motivated to choose $B$ unless enough other agents do to switch beyond the point $p=\theta$, the ratio of chooser $A$, which is known as a crucial mass parameter (threshold). It is enough merely to get 
agents to make the right choice at the beginning for the selection of $\mathrm{A}$ as collective efficiency.

López-Pintado and Watts (2005) studied the problem of spreading a particular behavior among agents located in a random social network. In each period of time, neighboring agents interact strategically playing the $2 \times 2$ coordination game shown in Table 1. Assuming myopic-best response dynamics, they showed that there exists a threshold for the degree of risk dominance of an action such that below the threshold, contagion of the action occurs. This threshold depends on the network's connectivity distribution. Based on this threshold concept, we show that the well-known scale-free networks (which are extremely popular in epidemiology) do not properly support this type of contagion, which is better accomplished by a more intermediate variance network.

López-Pintado and Watts also compared a homogeneous, an exponential and a scale-free random network with connectivity distributions $\mathrm{P}_{\mathrm{H}}(\mathrm{k}), \mathrm{P}_{\mathrm{E}}(\mathrm{k})$ and $\mathrm{P}_{\mathrm{SF}}(\mathrm{k})$, respectively. The exponential network had the highest threshold and the homogeneous network had the lowest, as follows:

$$
\theta_{H}^{*} \leq \theta_{S F}^{*} \leq \theta_{E}^{*}
$$

Notice that, as in the previous example, the network with an intermediate variance is the most successful for contagion. Also, the higher the average connectivity of the network, the lower the threshold. Again, the reason for this has to do with the existence of a giant component of vulnerable nodes. This result differs from the standard contagion models proposed to describe epidemic dynamics shown in the previous section.

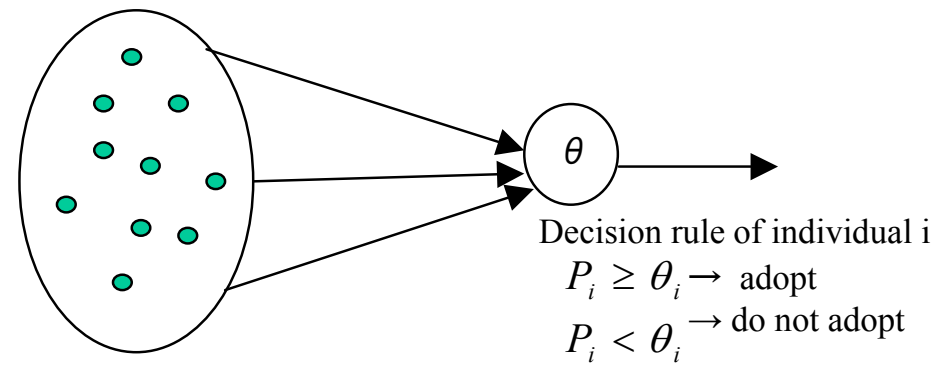

$\mathrm{Pi}$ : Ratio of neighbors who have already adopted

Figure 2. Basic scheme showing the spread of innovation

\section{Consensus and Synchronization on Social Networks}

Consensus problems have a long history in computer science and control theory. In networks of agents, consensus means to reach an agreement regarding a certain quantity of interest that depends on the state of all agents. A consensus algorithm is an interaction rule that specifies the information exchange between an agent and all of its neighbors on the network. The theoretical framework for solving consensus problems for networked systems was introduced by Olfati-Saber and colleagues (Olfati-Sabe, Fax \& Murray, 2007).

The analysis of consensus problems relies heavily on matrix theory and spectral 
graph theory. The interaction topology of a network of agents is represented using a directed graph $G$ with the set of nodes and edges. We denote neighbors of agent $i$ with $\mathrm{N}_{\mathrm{i}}$. Consider a network of agents with the following dynamics:

$$
\dot{x}_{i}(t)=\sum_{j \in N_{i}} a_{i j}\left(x_{j}(t)-x_{i}(t)\right)
$$

Here, reaching a consensus means asymptotically converging to the same internal state by way of an agreement characterized by the following equation:

$$
x_{1}=x_{2}=\ldots . .=x_{N}=\alpha
$$

Assuming that the underlying graph $G$ is undirected $\left(a_{i j}=a_{j i}\right.$ for all $\left.\mathrm{i}, \mathrm{j}\right)$, the collective dynamics converge to the average of the initial states of all agents:

$$
\alpha=\frac{1}{N} \sum_{i=1}^{N} x_{i}(0)
$$

The dynamics of system (6.1) can be expressed as

$$
\dot{\mathbf{x}}(t)=-\mathbf{L x}(t)
$$

$\mathrm{L}$ is the graph Laplacian of the network $\mathrm{G}$; the graph Laplacian is defined as

$$
\mathbf{L}=D-A
$$

where $\mathrm{D}=\operatorname{diag}\left(\mathrm{d}_{1}, \mathrm{~d}_{2, \ldots}, \mathrm{d}_{\mathrm{N}}\right)$ is the diagonal matrix with elements $\mathrm{d}_{\mathrm{i}}=\sum_{j \neq i} a_{i j}$ and $\mathrm{A}$ is the $\mathrm{N} \times \mathrm{N}$ matrix with elements $a_{i j}$ for all $\mathrm{i}, \mathrm{j}$.

We now consider directed networks where $a_{i j} \neq a_{j i}$ for some $\mathrm{i}, \mathrm{j}$. In this case, the underlying graph $G$ is called balanced if $\sum_{j \neq i} a_{i j}=\sum_{j \neq i} a_{j i}$ for all i. In a balanced graph, the total weight of edges entering a node (agent) and leaving the same node are equal for all nodes. Assuming that the underlying graph $G$ is directed but balanced, the collective dynamics in (6.1) converge to the average of the initial states of all agents as shown in (6.5).

A consensus protocol is an iterative method that provides the group with a common coordination variable. Network design problems for achieving faster consensus algorithms have attracted considerable attention from a number of researchers. The graph Laplacians and their spectral properties are important graph-related matrices that play a crucial role in the convergence analysis of consensus algorithms. The distributed consensus algorithm in (6.1) guarantees convergence to an agreement if the underlying network topology $\mathrm{G}$ is a connected graph; however, in a disconnected network, it is impossible for all nodes to reach an agreement.

It is worth mentioning that the second smallest eigenvalue $\lambda_{2}$ of the graph Laplacian L, called algebraic connectivity, quantifies the quality of the consensus algorithm. Therefore, network connectivity affects the performance and robustness properties of a system of networked agents. It is important for the agents to act in coordination with the other agents. To achieve coordination, individual agents do not need to share information; rather, they must be aware of the actions of other agents. In order to 
achieve a certain convergence and robustness in dynamical environment change, therefore, an appropriate network protocol must be designed. A consensus protocol is an interactive method that satisfies this need by providing the group with a common coordination variable.

A challenging issue is to analyze the diffusion process with a dynamic network topology that is time-varying, including subjects such as ad-hoc networks and synchronization of mobile agents. In the last several years, flocks of mobile agents have attracted significant attention (Vicsek, 1995). Efforts have been made to understand how a group of autonomous moving agents, such as flocks of birds or crowds of people, can form clustered groups without centralized coordination.

Such research is motivated by recent advances in communication and computation, as well as links to problems in social dynamics. Some problems have also been studied in ecology and theoretical biology, in the context of animal aggregation and social cohesion (Buchanam, 2007). The role of consensus algorithms in particle-based flocking is for an agent to achieve velocity matching with respect to its neighbors. Flocks are networks of dynamic systems with a dynamic topology. This topology is a proximity graph that depends on the state of all agents and is determined locally for each agent; i.e., the topology of flocks is a stated dependent graph.

It has been shown that flocking behavior results from a class of local control laws for the collection of agents. Flocking can occur when local action exploits the network properties of the underlying interconnections among agents. Exploiting modern results from algebraic graph theory, these properties are directly related to the topology of the network through the eigenvalues of the graph Laplacian; such an algorithm is possible using local control action agents that exploit the network properties of the underlying interconnection among agents. Network connectivity affects the performance and robustness properties of a system of networked agents.

One approach is to keep the weights fixed and design the topology of the network to achieve a relatively high degree of algebraic connectivity. A randomized algorithm for network design is proposed based on random rewiring that led to creation of the celebrated small-world model. The random rewiring of existing links of a network gives rise to considerably faster consensus algorithms, due to a multiple orders of magnitude increase in the algebraic connectivity of the network in comparison to a lattice type nearest-neighbor graph (Jackson \& Yariv, 2008).

A reasonable conjecture is that a small-world network should result in a good convergence speed for self-organization consensus problems due to its low average pair-wise path length, which should increase the speed of information diffusion in the system. This leads to a slight increase in the algebraic connectivity of the network, which is a measure of the speed of convergence of consensus algorithms (Motter, 2005).

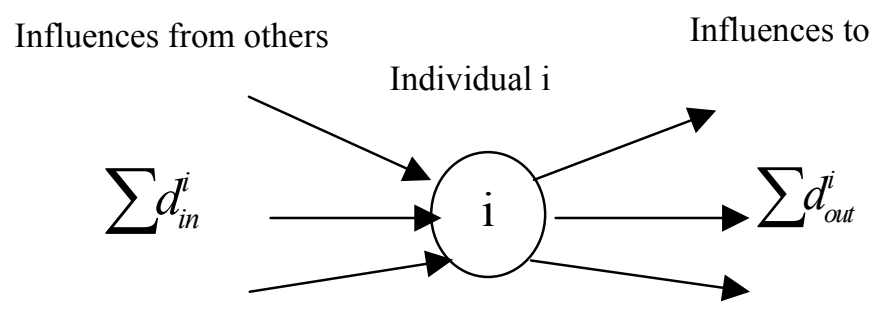

Figure 3. Basic scheme of consensus formation 


\section{Summary}

The diffusion process enhances an innovation via the feedback of information about the innovation's utility across different users; this feedback can then be used to improve the innovation. This aspect is similar to the micro-macro loop, which is an essential part of emergence. In this chapter, we provided an overview of research that examines how social network structure impacts emergent properties in diffusion processes. Most human social activities are substantially free of centralized management, and although people may care about the end result of the aggregate, individuals' decisions and behaviors are typically motivated by self interests. To make the connection between microscopic behavior and macroscopic patterns of interest, we should look at the system of interactions among agents - this can be described as the interaction topology. We focused on the stylized facts of macroscopic emergent phenomena that are results of the bi-directional interactions. Social network topologies determine a basic and important form of social interactions among agents, and microscopic behaviors of agents largely determine the diffusion patterns observed at the macroscopic level.

Many diffusion processes are progressive in the sense that once a node switches from one state A to another state B, it remains with B in all subsequent time steps. It was shown that scale-free networks with hub nodes foster diffusion. The other type of diffusion is a non-progressive process in which, as time progresses, nodes can switch from state A to B or from B to A, depending on the states their neighbors. In the case of a non-progressive process such as innovation, diffusion processes largely determine individual decisions. In this case, exponential networks with many midsize hub nodes foster diffusion, as demonstrated by the epidemic and rumor models. We also gave a brief overview of the consensus and synchronization processes that relate to the emergence of coherent patterns by emphasizing the underlying network topology. In this case, a network with a balanced underling network topology is necessary for the emergence of coordination.

As a result, we now have a better understanding of the emergent properties of desirable aggregate process. How humans act is often influenced by friends, acquaintances or neighbors - social networks, in other words, are an important form of social interaction. We conclude that topological properties of networks should be derived from the dynamics from which the networks were created. A large number of interactions is not enough to guarantee emergence. In some cases, the diffusion process may need to reach a certain critical mass - a combined threshold of diversity, organization and connectivity_-before desirable emergent properties appear.

\section{References}

Arthur, W. (1989). Competing Technologies, Increasing Returns, and Lock-In By Historical Events. Economic Journal 99, 116-131.

Ball, P. (2004). Critical Mass, Farrar, Straus and GirouxBanerjee, A. (1992). A Simple Model of Herd Behavior. Quarterly Journal of Economics 110, 797-817.

Banerjee, A. (1992). A Simple Model of Herd Behavior, Quarterly Journal of Economics, 107, 797-817.

Barabási, A., Albert, R., Jeong, H. (2000). Scale-free characteristics of random networks: the topology of the World-Wide Web. Physica A. 281, 69-77.

Bass, F.M.(1969). A New Product Growth Model for Consumer Durables, Management Science, 13(5), 215-227 
Boyd, S., Ghosh, A. Prabhakar, B., Shah, D. (2006). Randomized Gossip Algorithms.

I EEE TON 14, Issue SI, 2508-2530.

Buchanam, M. (2007). The Social Atoms, Reed Elsevier, Inc. Chakravorti, B. (2003). The Slow Pace of Fast Change: Bringing Innovations to Market in a Connected World. Boston, Harvard Business School Press.

Chatterjee, K.,Susan,H. (2004). Technology Diffusion by Learning from Neighbors, Advances in Applied Probability, 36, 355-376.

Colizza, B, Barrat, A., Barthelemy, M., and Vespignamic, D. (2006). The role of the airline transportation network in the prediction and predictability of global epidemics, PNAS 103 2015-2020.

Durrent, R. (2007). Random Graph Dynamics, Cambridge University Press[

Ellison, G. (1993). Learning, Local Interaction, and Coordination. Econometrica 61 (5), 1047-1071.

López-Pintado, D., Watts, D.J. (2005). Social influence and random networks. Mimeo, Columbia University

Hall, B. (2004). Innovation and Diffusion, Handbook of Innovation, In Fagerberg, J., D. Mowery, and R. R. Nelson (eds.), Oxford University Press.

Immorica, N., Kleinberg, M, Mahdian, M. (2007). The Role of Compatibility in the Diffusion of Technologies through Social Networks, The Proceedings of Electronic Commerces (EC'07)

Jackson, M., Yariv, L. (2008). Diffusion, Strategic Interaction and Social Structure, TR-Stanford University.

Katona, Z., Zubcsek, P., Sarvary, M. (2007). Joining the Network: Personal Influences as Determinants of Diffusion, TR, UC Berkeley

Meyers, L., Pourbohloul D, Newman, M.E.J. (2005). Network Theory and SARS: prediciting outbreak diversity. Journal of Theoretical Biology 232, 71-81.

Lopetz-Pintado, D. (2006). Contagion and coordination in random networks. Int. Journal of Game Theory, 34, 371-382.

Morris, S. (2000). Contagion. Review of Economic Studies 67, 57-78J.

Motter, A., Zhou, C., Kutth, J. (2005). Network synchronization, Diffusion, and the Paradox of Heterogeneity, Physical Review E. 71, 016116-1-9.

Namatame, A. (2006). Adaptation and Evolution in Collective Systems, World Scientific

Newman, M.E.J. (2003). The structure and function of complex networks. Society for Industrial and Applied Mathematics, 45(2), 167-256.

Nowak, M. (2006). Evolutionary Dynamics, Harvard University Press

Olfati-Saber, R., Fax, J.A., Murray, R.M. (2007). Consensus and Cooperation in Networked Multi-Agent Systems. Proceedings of the IEEE, 95(Jan). 401-420.

Reynolds, C. (1987). Flocks, Herds, and Schools: A Distributed Behavioral Model. Computer. Graph. 21, 25 http://www.red3d.com/cwr/papers/1987/boids.html

Rogers, E., Media, U., Rivera, M., Wiley, C. (2005). Complex Adaptive Systems and The Diffusion of Innovations, The Innovation Journal ,10(3), 1-26.

Rosenberg, N. (1972). Factors Affecting the Diffusion of Technology, Explorations in Economic History, 10(1). 3-33

Spielman, D.J. (2005). Systems of Innovation: Models, methods, and future directions. Innovation Strategy Today 2(1), 55-66.

Sole, R., Ferrrer-Cancho,P, Montota, R. (2003). Selection, Tinkering, and Emergence in 
Complex Networks. Complexity 8(1), 20-33.

Wang, Y., Chakrabarti, D, (2003). Epidemic Spreading in real Networks: An Eigenvalue Viewpoint. Proc. of 22nd Symposium on Reliable Distributed Computing, pp.242-262.

Watts, D. (2007). Influentials, Networks, and Public Opinion Formation, Journal of Consumer Research

Watts, D. (1999) Small World. The Princeton University Press.

Vicsek, T. (1995). Novel Type of Phase Transition in a System of Self-Driven Particles. Phys. Rev. Lett. 75, 1226.

Young, P. (2007) Innovation Diffusion in Heterogeneous Populations: Contagion, Social Influence, and Social Learning 